\title{
Coulomb drag in graphene single layers separated by thin spacer
}

\author{
M. I. Katsnelson ${ }^{1}$ \\ ${ }^{1}$ Radboud University of Nijmegen, Institute for Molecules and Materials, \\ Heyendaalseweg 135, NL-6525 AJ Nijmegen, The Netherlands
}

(Dated: October 14, 2018)

\begin{abstract}
Motivated by very recent studies of Coulomb drag in grahene-BN-graphene system we develop a theory of Coulomb drag for the Fermi liquid regime, for the case when the ratio of spacer thickness $d$ to the Fermi wavelength of electrons is arbitrary. The concentration $(n)$ and thickness dependence of the drag resistivity is changed from $n^{-3} d^{-4}$ for the thick spacer to $n^{-1}\left|\ln \left(n d^{2}\right)\right|$ for the thin one.

PACS numbers: 72.80.Vp, 73.21.Ac, 73.63.Bd
\end{abstract}

Coulomb drag in bilayer semiconductor systems is a very interesting phenomenon providing a unique information about many-body effects $\underline{\underline{1}} \underline{\underline{4}}$. Since the role of electron-electron interactions in graphene, a novel twodimensional material with extraordinary electronic and structural properties ${ }^{5-9}$, is a controversial issue now (for review, see Ref.10) study of the Coulomb drag in graphene is important, as a way to clarify the situation.

First theoretical ${ }^{11,12}$ and experimental ${ }^{13,14}$ studies of the Coulomb drag in graphene have been performed already. Theory 11 deals with the case of thick spacer $\left(k_{F} d \gg 1\right.$, where $k_{F}$ is the Fermi wave vector of graphene and $d$ is the spacer thickness), and the results are in a good agreement with the corresponding experimental data $^{13}$ (the effects of trigonal warping ${ }^{12}$ seem to be negligible). Very recently, the group of A. Geim and K. Novoselov has performed experiments with graphene on a substrate of $\mathrm{BN}$, using also $\mathrm{BN}$ as a spacer between two graphene single layers, and observed the drag for much thinner spacers 14 . Here I present a theory generalizing that of Ref 11 for arbitrary values of $k_{F} d$.

We start with the general expression for the drag conductivity 1,2 based on the lowest-order perturbation theory in interlayer Coulomb interaction (we consider only the case of identical layers 1 and 2, qualitatively all the basic physics remains the same for the case of different doping of two layers):

$\sigma_{D}=\frac{1}{16 \pi k_{B} T} \sum_{\mathbf{q}} \int_{-\infty}^{\infty} \frac{d \omega}{\sinh ^{2}\left(\frac{\hbar \omega}{2 k_{B} T}\right)} \Gamma_{x}^{2}(\mathbf{q}, \omega)\left|U_{12}(q, \omega)\right|^{2}$

where $\Gamma_{x}$ is the nonlinear susceptibility of electrons in the layer, $E_{F}$ is their Fermi energy, $T$ is the temperature, $U_{12}$ is the Fourier component of the screened interlayer Coulomb interaction which reads, for the case of identical layers:

$$
U_{12}(q, \omega)=\frac{u_{c}(q)}{\left[1+v_{c}(q) \Pi(q, \omega)\right]^{2}-\left[u_{c}(q) \Pi(q, \omega)\right]^{2}},
$$

$v_{c}(q)$ and $u_{c}(q)$ are the Fourier components of bare Coulomb interactions within the layer and between the layers, respectively, $\Pi(q, \omega)$ is the polarization operator of the electron gas in graphene. In vacuum, $v_{c}(q)=$ $2 \pi e^{2} / q$ and $u_{c}(q)=v_{c}(q) \exp (-q d)$ and the expression (2) coincides with Eq.(A2) from Ref.1.

Since typical frequencies contributing to the integral in Eq.(1) is of the order of $k_{B} T / \hbar$ one can assume, for low enough temperatures, that the screening is static and replace $U_{12}(q, \omega)$ by $U_{12}(q, 0)$.

In the ballistic regime (which means that the distance between the layers $d$ is much smaller than the mean-free path within the layer $l$ ) one can calculate for the case of graphene $\underline{\underline{11}}$

$$
\Gamma_{x}(\mathbf{q}, \omega) \approx-\frac{4 e \tau \omega}{\pi v} \frac{q_{x}}{q}
$$

at $\omega \rightarrow 0$, where $\tau=l / v$ is the mean-free path time and $v \approx 10^{6} \mathrm{~m} / \mathrm{s}$ is the Fermi velocity.

Assuming that $\sigma_{D} \ll \sigma$ where $\sigma$ is the inlayer conductivity the drag resistivity is $\rho_{D}=-\sigma_{D} / \sigma^{2}$. Drude formula for the case of graphene is

$$
\sigma=\frac{e^{2}}{\pi \hbar^{2}} E_{F} \tau
$$

thus, the drag resistivity does not depend on $\tau$.

As a result, the drag resistivity for the case of identical graphene layers takes the form

$$
\rho_{D}=-\frac{2 h}{3 e^{2}}\left(\frac{k_{B} T}{E_{F}}\right)^{2} \sum_{\mathbf{q}}\left|\frac{U_{12}(q, 0)}{\hbar v}\right|^{2}
$$

The quantity $U_{12}$ in Eq.(15) is given by the expression (2) with the static polarization operator for graphene (see, e.g., Refs, 15, 16) 


$$
\Pi(q, 0)=\left\{\begin{array}{cc}
\frac{2 k_{F}}{\pi \hbar v}, & q<2 k_{F} \\
\frac{2 k_{F}}{\pi \hbar v}+\frac{q}{2 \pi \hbar v}\left[\cos ^{-1} \frac{2 k_{F}}{q}-\frac{2 k_{F}}{q} \sqrt{1-\left(\frac{2 k_{F}}{q}\right)^{2}}\right], & q>2 k_{F}
\end{array}\right.
$$

To find $v_{c}(q)$ and $u_{c}(q)$ one needs to solve the electrostatic problem taking into account different screening by substrate, spacer, and air. Let us assume that the dielectric medium is three-layer, with the dielectric constant distribution

$$
\varepsilon=\left\{\begin{array}{l}
\varepsilon_{1}, \quad z>d \\
\varepsilon_{2}, \quad d>z>0 \\
\varepsilon_{3}, \quad z<0
\end{array}\right.
$$

The calculations are quite standard (see, e.g., Ref.17). However, for the reader's convenience we present them here with some details.

We have to solve the Poisson equation

$$
\frac{d}{d z}\left(\varepsilon(z) \frac{d \varphi(z)}{d z}\right)-q^{2} \varepsilon(z) \varphi(z)=-4 \pi e \delta(z-\eta)
$$

where $\varphi(z)$ is the electrostatic potential created by the point charge $e$ situated at $x=0, y=0, z=\eta \rightarrow+0$. The only allowed solution at $z<0$ is

$$
\varphi(z)=A e^{q z}
$$

and at $z>d$ is

$$
\varphi(z)=B e^{-q z}
$$

For $\eta<z<d$ it should be tried in the most general form:

$$
\varphi(z)=\alpha e^{q z}+\beta e^{-q z}
$$

From continuity of the potential and the normal component of electric induction, $D_{n}=-\varepsilon \frac{d \varphi}{d z}$ at the boundaries $z=0$ and $z=d$ we find, taking into account Eqs. (9) and (10):

$$
\begin{aligned}
& \frac{\varphi^{\prime}(-0)}{\varphi(-0)}=\frac{\varepsilon_{3}}{\varepsilon_{2}} q, \\
& \frac{\varphi^{\prime}(d)}{\varphi(d)}=-\frac{\varepsilon_{1}}{\varepsilon_{2}} q
\end{aligned}
$$

where prime means the derivative with respect to $z$. The potential $\varphi(z)$ is continuous at $z=\eta(\varphi(-0)=\varphi(+0))$ but its derivative has a jump. Due to Eqs. (8), (9), (12)

$$
\varphi^{\prime}(+0)=\frac{\varepsilon_{3}}{\varepsilon_{2}} q A-\frac{4 \pi e}{\varepsilon_{2}}
$$

At last, we can find from Eqs. (14) and (13) the constants $\alpha$ and $\beta$. The final answer for $v_{c}(q)=e \varphi(z=0)$ and $u_{c}(q)=e \varphi(z=d)$ reads:

$$
\begin{aligned}
& u_{c}(q)=\frac{8 \pi e^{2} \varepsilon_{2} \exp (q d)}{q\left[\left(\varepsilon_{1}+\varepsilon_{2}\right)\left(\varepsilon_{3}+\varepsilon_{2}\right) \exp (2 q d)-\left(\varepsilon_{1}-\varepsilon_{2}\right)\left(\varepsilon_{3}-\varepsilon_{2}\right)\right]} \\
& v_{c}(q)=\frac{8 \pi e^{2} \varepsilon_{2} \exp (q d)\left[\varepsilon_{2} \cosh (q d)+\varepsilon_{1} \sinh (q d)\right]}{q\left[\left(\varepsilon_{1}+\varepsilon_{2}\right)\left(\varepsilon_{3}+\varepsilon_{2}\right) \exp (2 q d)-\left(\varepsilon_{1}-\varepsilon_{2}\right)\left(\varepsilon_{3}-\varepsilon_{2}\right)\right]}
\end{aligned}
$$

For simplicity, we will consider further only the case $\varepsilon_{1}=\varepsilon_{2}$ (which, actually, takes place in the experimental situation ${ }^{14}$ where $\mathrm{BN}$ is used both as a substrate and as a spacer). In this case, the expression (15) is simplified dramatically:

$$
\begin{aligned}
& u_{c}(q)=v_{c}(q) \exp (-q d), \\
& v_{c}(q)=\frac{4 \pi e^{2}}{q\left(\varepsilon_{2}+\varepsilon_{3}\right)}
\end{aligned}
$$

and

$$
U_{12}=\frac{v_{c}}{2\left(v_{c} \Pi\right)^{2} \sinh (q d)+\left(1+2 v_{c} \Pi\right) \exp (q d)} .
$$

Substituting Eqs.(6), (15), (17) into Eq.(5) we have:

$$
\rho_{D}=-\frac{h}{e^{2}} \frac{\pi}{48}\left(\frac{k_{B} T}{E_{F}}\right)^{2} \frac{1}{\alpha^{2}} F\left(2 k_{F} d\right)
$$

where

$$
\alpha=\frac{2 e^{2}}{\hbar v\left(\varepsilon_{2}+\varepsilon_{3}\right)}
$$

is the effective "fine structure" constant (for the combination of $\mathrm{BN}$ with $\varepsilon_{2} \approx 4$ and air with $\varepsilon_{3}=1$ we have 


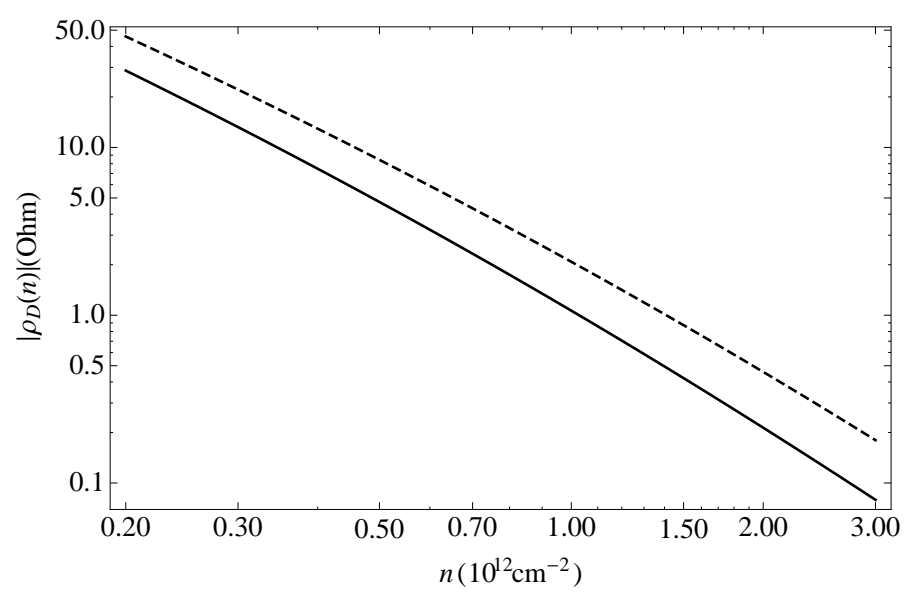

FIG. 1: Drag resistivity (18) as a function of charge carrier concentration, for $\varepsilon_{3}=1, \varepsilon_{2}=4, T=120 \mathrm{~K}, d=3 \mathrm{~nm}$ (dashed line) and $d=4 \mathrm{~nm}$ (solid line)

$\alpha \approx 0.87$ ) and the function $F$ is represented as

$$
F(y)=\int_{0}^{\infty} d x \frac{x^{3}}{\left[\varphi^{2}(x) \sinh (y x)+\frac{x(x+4 \alpha \varphi(x))}{8 \alpha^{2}} \exp (y x)\right]^{2}}
$$

where

$$
\varphi(x)=\left\{\begin{array}{cc}
1, & x<1 \\
1+\frac{x}{2}\left(\cos ^{-1} \frac{1}{x}-\frac{\sqrt{x^{2}-1}}{x^{2}}\right), & x>1
\end{array}\right.
$$

In the limit $y \gg 1$

$$
F(y) \cong \frac{3 \zeta(3)}{2 y^{4}}
$$

and Eqs.(18), (20) give the known result ${ }^{11}$

$$
\rho_{D}=-\frac{h}{e^{2}} \frac{\pi \zeta(3)}{32}\left(\frac{k_{B} T}{E_{F}}\right)^{2} \frac{1}{\left(k_{F} d\right)^{2}} \frac{1}{(\kappa d)^{2}}
$$

where $\kappa=4 \alpha k_{F}$ is the inverse screening radius.

In the opposite limit $y \ll 1$ typical values of $x \approx 1 / y \gg$ 1 and one can assume $\phi(x) \approx \pi x / 4$ which gives

$$
F(y) \cong\left(\frac{8 \alpha^{2}}{1+\pi \alpha}\right)^{2} \ln \frac{1}{y}
$$

The behavior of drag resistivity as a function of charge carrier concentration for $k_{F} d$ of the order of one is shown in Fig. 1. This result seems to be in a qualitative agreement with the experimental data $\frac{14}{}$, at least, it gives the correct order of magnitude for the drag resistivity. At the same time, for small enough $k_{F} d$ and $\alpha \approx 1$ the interlayer Coulomb interaction is in general not small, and it is not clear whether the lowest-order perturbation theory used here will be also quantitatively accurate or taking into account next-order contributions will be necessary. The issue requires further studies, both experimental and theoretical.

Recently two more works on the subject appeared $\underline{18,19}$. The results of this paper and the other two papers concerning concentration dependence of the drag resistivity are in an agreement, namely, in Ref 18 the same analytical concentration dependence as here, $\rho_{D} \propto$ $n^{-1}\left|\ln \left(n d^{2}\right)\right|$, was obtained for the case of thin spacer whereas in Ref 19 the numerical data were fitted by $\rho_{D} \propto n^{-\alpha}$ with $\alpha$ of the order of one.

\section{Acknowledgement}

I am thankful to Andre Geim for stimulating discussions of unpublished experimental results 14 and to Timur Tudorovskiy for helpful discussions. This work is part of the research program of the Stichting voor Fundamenteel Onderzoek der Materie (FOM), which is financially supported by the Nederlandse Organisatie voor Wetenschappelijk Onderzoek (NWO).
1 A. Kamenev and Y. Oreg, Phys. Rev. B 52, 7516 (1995).

${ }^{2}$ K. Flensberg, Ben Yu-Kuang Hu, A.-P. Jauho, and J. M. Kinaret, Phys. Rev. B 52, 14761 (1995).

${ }^{3}$ G. Vignale and A. H. MacDonald, Phys. Rev. Lett. 76, 2786 (1996).

4 J. A. Seamons, C. P. Morath, J. L. Reno, and M. P. Lilly, Phys. Rev. Lett. 102, 026804 (2009).

5 A. K. Geim and K. S. Novoselov, Nature Mater. 6, 183 (2007).

6 M. I. Katsnelson, Mater. Today 10, 20 (2007).

7 A. H. Castro Neto, F. Guinea, N. M. R. Peres, K. S. Novoselov, and A. K. Geim, Rev. Mod. Phys. 81, 109 (2009).

8 A. K. Geim, Science 324, 1530 (2009).

${ }^{9}$ M. A. H. Vozmediano, M. I. Katsnelson, and F. Guinea,
Phys. Rep. 496, 109 (2010).

${ }^{10}$ V. N. Kotov, B. Uchoa, V. M. Pereira, A. H. Castro Neto, and F. Guinea, arXiv:1012.3484.

11 W.-K. Tse, Ben Yu-Kuang Hu, and S. Das Sarma, Phys. Rev. B 76, 081401 (2007).

12 B. N. Narozhny, Phys. Rev. B 76, 153409 (2007).

13 S. Kim, I. Jo, J. Nah, Z. Yao, S. K. Banerjee, and E. Tutuc, Phys. Rev. B 83, 161401 (2011).

14 A. K. Geim, private communication; R. V. Gorbachev, talk at Graphene Week (Obergurgl, April 2011).

15 T. Ando, J. Phys. Soc. Japan 75, 074716 (2006).

16 B. Wunsch, T. Stauber, F. Sols, and F. Guinea, New J. Phys. 8, 318 (2006).

17 R. E. V. Profumo, M. Polini, R. Asgari, R. Fazio, and A. H. MacDonald, Phys. Rev. B 82, 085443 (2010). 
18 E. H. Hwang and S. Das Sarma, arXiv:1105.3203.

Castro Neto, Europhys. Lett. 95, 18001 (2011).

19 N. M. R. Peres, J. M. B. Lopes dos Santos, and A. H. 